\title{
Assignment of chiral indices of Boron Nitride Nanotubes by Electron Diffraction
}

\author{
R. Arenal *, M. Kociak**, A. Loiseau***, D.J. Miller* \\ * EMC group, MSD Argonne National Laboratory, IL 60439 Argonne - USA \\ ** LPS, Université Paris-Sud and CNRS, 91405 Orsay - France \\ *** LEM, UMR 104 CNRS-ONERA, 92322 Châtillon - France
}

Boron nitride nanotubes (BNNTs) are an emerging nanomaterial with properties complementary to those of the well-known carbon nanotubes. BN tubes are insulators with a constant band gap larger than $5.5 \mathrm{eV}$, independent of their helicity and diameters [1]. These tubes can be used for high-powerhigh-frequency electronic devices; they also have outstanding mechanical properties, are chemically inert, and can operate as protective cages in the nanoworld. In comparison with single wall carbon nanotubes (SW-CNTs), relatively little is known about the growth of BNNTs. A detailed knowledge of the atomic structure of these nanotubes (chiral angle and diameter) is fundamental to understanding their growth mechanism. The most reliable technique for obtaining this information is electron diffraction. We recorded the intensities of electron diffraction patterns (EDPs) from individual SW-BNNTs and multi-walled (MW) nanotubes as well as from bundles of BNNTs [2]. This work represents the first determination of the chiral indices of SW- and double-walled (DW-) BNNTs. Furthermore, the analysis of more than 100 NTs provides to us an accurate helicity distribution of these tubes.

BNNTs were grown using the laser vaporization technique described in $[3,4]$. A high percentage of the tubes are SW (isolated and forming small bundles) and some are MW-BNNTs (mainly doublewalled). In order to avoid the unwanted contribution of another tube, we used the nano beam electron diffraction technique. We performed this work in a FEI Tecnai F20 transmission electron microscope operating at $100 \mathrm{keV}$ and equipped with a field-emission gun (FEG). We employed the smallest condenser aperture, providing a highly coherent and parallel nanoprobe $(40-50 \mathrm{~nm})$. The EDPs of the NTs were recorded using a charge-coupled device (CCD) camera. We note that no "blooming" effect was observable. The acquisition time of each EDPs was less than 10 seconds. We have checked that with this procedure, several EDPs can be acquired without any radiation damage of the tube.

We measured the chiral angles of all of the tubes (individual and ropes) inspected and also obtained the diameter of most of the individual tubes. In the case of individual SW and DW tubes, their chiral indices were determined as the best value fitting experimental EDPs and simulated EDPs calculated using kinematical electron theory [5]. Fig. 1 (a) shows the bright field image of a SW-BNNT acquired under nanoprobe illumination. Fig. 1 (b) corresponds to the EDPs of this tube. The EDPs of a SW NT consist of a set of lines perpendicular to the tubule axis direction known as layer-lines [5]. The central one is called the equatorial line (Eq-L). The helicity $(\alpha)$ of the tube is determined by measuring the interspacing between the different layers-lines [6]. In the case of this SW-BNNT (Fig. 1 ), $\alpha=2.01^{0}$. The tube diameter (d) is extracted from the oscillations of the Eq-L. We found $\mathrm{d}=$ $2.36 \mathrm{~nm}$. With these parameters, we simulate the corresponding EDPs and determined the chiral indices as the best fit between experimental and simulated EDPs. The SW-BNNT of Fig. 1 is thus unambiguously identified as a $(29,1)$. Fig. 2 (a) displays the EDPs of an isolated DW-BNNT. For a DW NT, the assignment of the chiral indices is more complex due to the superimposition of the 
EDPs of inner and outer tubes but is done following the same procedure [7]. By measuring the interlayer distance we found that the $\alpha$ of these tubes is $18.7^{0}$ and $25.6^{\circ}$, respectively. The pseudooscillations of the Eq-L provide values of the mean diameter and of the inter-tube distance equal to $2.68 \mathrm{~nm}$ and $0.39 \mathrm{~nm}$, respectively. Finally, chiral indices of this tube are found to be $(23,11) @(26,20)$. Fig. 2 (b) shows the corresponding simulated EDPs.

The main conclusion of thus systematic study is that BNNTs show a clear tendency to the zig-zag configuration whereas other helicities are randomly distributed. This tendency is consistent with observations of these tubes in high resolution imaging mode [3], where tubes with zig-zag configuration could be easily found. This structural property makes BNNTs different from carbon analogs and will be discussed.

\section{References}

[1] R. Arenal, et al., Phys. Rev. Lett. 95, 127601 (2005).

[2] R. Arenal, M. Kociak, A. Loiseau, D.J. Miller, submitted (2006).

[3] R.S. Lee et. al., Phys. Rev. B, 64, 121405(R) (2001).

[4] R. Arenal, J.L. Cochon, O. Stephan, A. Loiseau, in preparation.

[5] P. Lambin, A. Lucas, Phys. Rev. B 56, 3571 (1997).

[6] M. Gao et al., Appl. Phys. Lett. 82, 2703 (2003).

[7] M. Kociak, K. Hirahara, K. Suenaga, S. Iijima, Eur. Phys. J. B 32, 405 (2003).

[8] This work was supported in part by the U.S. DOE under BES-MS W-31-109-Eng-38 at ANL

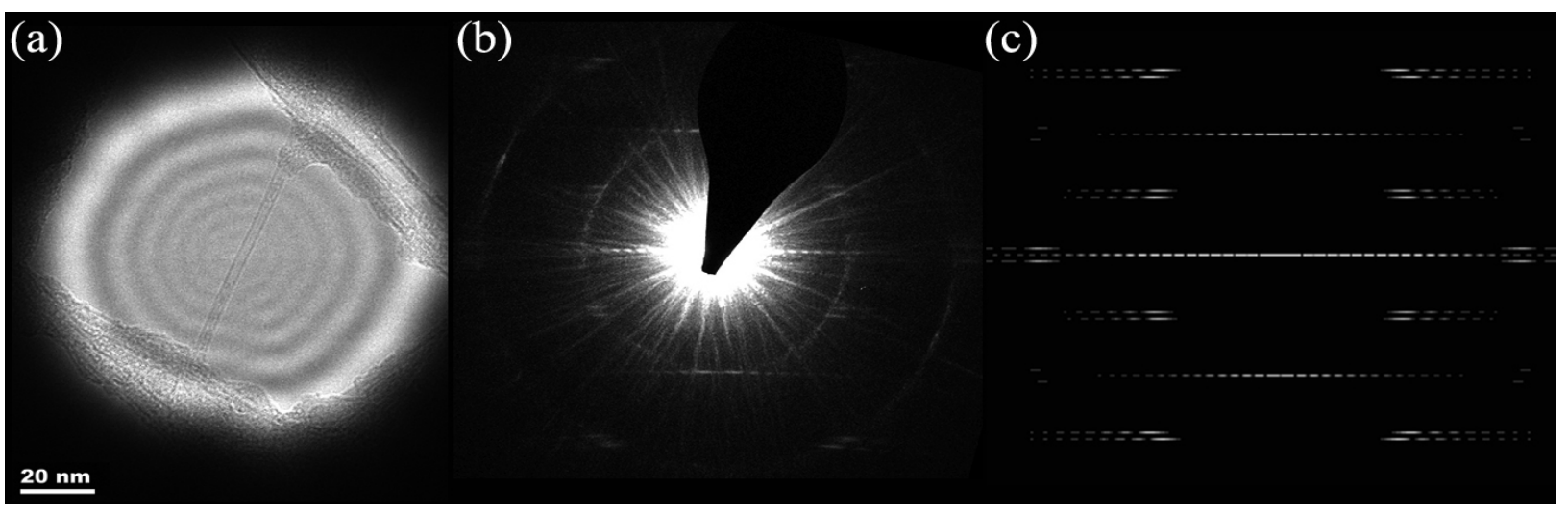

Figure 1. (a) Bright field image of a SW-BNNT. (b) and (c) experimental and simulated EDPs of this SW-BNNT, respectively. We assign these EDPs unambiguously to a $(29,1)$ NT.

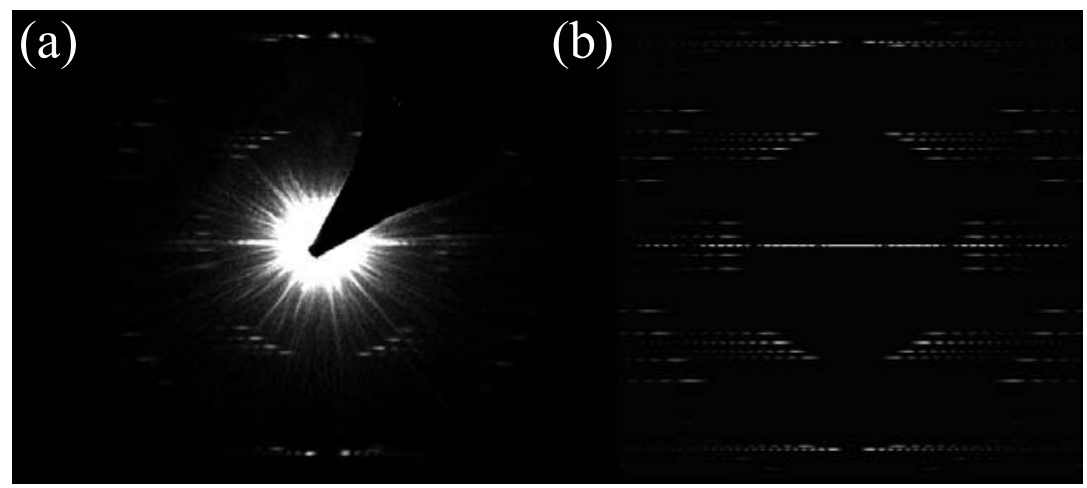

Figure 2. (a) Experimental EDPs of a DW-BNNT. (b) Simulated EDPs of this DW-BNNT, corresponding to a $(23,11) @(26,20)$ DW-BNNT. 